\title{
Cat Days
}

\section{Matt Hetherington}

When you only lie around yawning, dozing, \& licking yourself

when you cast a clear eye

on death \& taxing activities

when you abide where the warmth is, tasting the soft food one mouthful at a time

\& where time is merely a habit you had once when young and unwise yet still the night waits like a purring jungle. 